\title{
Personalized hepatitis C therapy: opportunities and pitfalls
}

\author{
Expert Rev. Mol. Diagn. 11(2), 127-129 (2011)
}

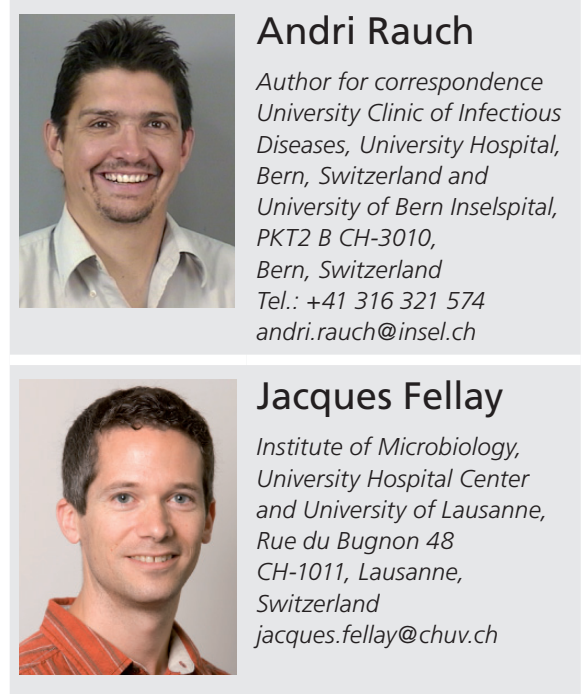

EXPERT
REVIEWS

\begin{abstract}
"Recent discoveries in the human genomics of hepatitis $C$ virus infection open new avenues in basic science and substantially improve our understanding of the natural history of the disease and of treatment outcomes."
\end{abstract}

Experienced physicians have always used personalized therapy in clinical routine, long before the term personalized medicine was introduced. Optimal patient care is based on careful selection of patients who need treatment, and of therapeutic regimens that are likely to be effective and well tolerated. The clinical management of patients suffering from chronic hepatitis $\mathrm{C}$ virus $(\mathrm{HCV})$ infection represents an ideal setting to demonstrate the relevance of personalized medicine, the intricate roles of genetic and nongenetic factors, as well as the opportunities and risks associated with individualization of patient care. In the context of chronic HCV infection, the key questions are: 'Who needs therapy?', 'Will therapy be effective?' and 'Will therapy be tolerated?'

\section{Who needs therapy?}

The progression of liver disease caused by chronic HCV infection is extremely variable. Some individuals will never develop clinical disease, while others will die from liver-related complications within a few years if left untreated. Risk factors that have been consistently associated with faster progression of liver disease include older age at infection, male gender, alcohol intake and co-infection with HIV or hepatitis $B$ virus [1]. However, none of these factors are sufficient to replace a careful assessment of liver disease by either liver biopsy or noninvasive measurement of liver disease. Accordingly, international guidelines uniformly recommend an assessment of liver disease prior to starting
HCV therapy. Estimation of the current stage of liver disease by histological analysis of biopsy material or by noninvasive markers of liver fibrosis is clearly more important for counseling patients than demographic, clinical or genetic predictors of fibrosis. However, it remains to be established whether the addition of these predictors could help further improve patient care. For example, individuals with moderate fibrosis could be managed according to their individual risk assessment, with more urgent treatment or closer follow-up proposed to patients with high-risk profiles. Currently, even if some risk factors are already taken into account by treating physicians and occasionally 'tip the balance' for or against therapy in difficult cases, the response to the question 'Who needs therapy?' still largely depends on the assessment of liver fibrosis.

"The clinical management of patients suffering from chronic hepatitis $C$ virus infection represents an ideal setting to demonstrate the relevance of personalized medicine..."

\section{Will therapy be effective?}

It has long been known that viral and host factors strongly influence the response to HCV treatment. Until recently, the viral genotype was the strongest predictor of response to HCV therapy with pegylated interferon and ribavirin (peg-IFN/RBV). 
Permanent viral eradication can be achieved in up to $80 \%$ of individuals infected with 'favorable' HCV genotypes (2 or 3), but only in approximately $40 \%$ of those infected with 'unfavorable' HCV genotypes (1 or 4) [2]. In addition, response rates are higher in females, in those with lower HCV RNA levels, and in Asians and Caucasians, compared with individuals of recent African ancestry [2]. The contribution of human genetic variation to treatment response, although suspected, could not be convincingly demonstrated before last year, when several genomewide association studies reported a major breakthrough in HCV genomics. These studies identified variation in the IL28B gene region as a major determinant of HCV treatment response [3-7]. Shortly thereafter, the same genetic factor was also shown to be the strongest predictor of natural HCV clearance [6,8]. In HCV genotype 1 infection, IL28B polymorphism was the strongest predictor of treatment response, independent of other known factors, and also predicted viral kinetics during HCV therapy [9]. IL28B encodes IFN- $\lambda 3$ (type III IFN), a cytokine with antiviral activity. Additional studies demonstrated that protective $I L 28 B$ genotypes associate with lower pretreatment levels of interferonstimulated genes [10], a finding in line with previous observations that baseline upregulation of interferon-stimulated genes impairs the response to HCV therapy [11]. The association of IL28B variants with response to HCV therapy was replicated in numerous studies, was consistent between ethnicities, and was independent of co-infection with HIV (reviewed in [7]). Interestingly, in transplanted individuals, both donor and recipient IL28B genotypes were associated with response to HCV therapy [12]. While there is overwhelming evidence for the role of $I L 28 B$ variation in the host response to HCV genotype 1 or 4 infection, it is uncertain whether $I L 28 B$ variation has a major impact on treatment response in those infected with 'easy-to-treat' genotypes (HCV genotypes 2 or 3) [6].

Predicting treatment efficacy is a difficult task in routine clinical practice. Multiple clinical and laboratory parameters need to be considered before and during HCV therapy. The addition of IL28B genotypic information can substantially improve the prediction of treatment outcomes and can, in some instances, help patients and physicians to make more informed decisions regarding HCV therapy [13]. For example, genotypic information can identify individuals with very low probability of treatment response, and thereby avoid potentially harmful side effects of an ineffective treatment. IL28B genotypic information can also predict HCV eradication during treatment, which can help to estimate whether continuing therapy outweighs the risk of side effects [9].

The role of $I L 28 B$ genotyping in the coming era of combination therapy, associating pegIFN/RBV and protease or polymerase inhibitors, has yet to be fully determined. However, as long as IFN is included in the treatment regimen, IL28B variation can be expected to have a detectable impact, unless the success rate approaches $100 \%$. This has recently been demonstrated in a cohort of Japanese patients treated with peg-IFN/RBV and the protease inhibitor telaprevir, in which IL28B genotype was highly associated with treatment response [14].
Finally, IL28B genotyping certainly has a place as a randomization criterion in early-stage clinical trials of anti-HCV combinations that include peg-IFN: any imbalance in the distribution of IL28B genotypes between study arms would substantially confound the results of small-scale trials [15].

\section{Will therapy be tolerated?}

Tolerability is a major issue during therapy with peg-IFN/RBV. In routine clinical settings, a substantial proportion of individuals do not start HCV treatment owing to fear of adverse events [16]. In addition, up to $25 \%$ of treated individuals stop therapy early owing to side effects [17]. In many cases, peg-IFN and/or RBV doses need to be reduced, which reduces sustained virological response rates. Patients are routinely screened for comorbidities that could represent contraindications to $\mathrm{HCV}$ treatment, but there is otherwise no direct way to predict the occurrence of any severe toxicity. Recently, a genome-wide association approach was successfully used in the search for determinants of drug-induced anemia, which occurs in most treated patients and is essentially attributable to RBVassociated red blood cell destruction: genetic variants associated with differences in susceptibility to anemia were identified in the inosine triphosphatase (ITPA) gene region [18,19]. Interestingly, the association signal was entirely explained by two functional polymorphisms that cause ITPA deficiency, a benign enzymopathy that turns out to play a significant protective role in the particular context of HCV treatment. This finding has the potential to shed new light on RBV metabolism and mode of action.

\section{"The addition of IL28B genotypic information can substantially improve the prediction of treatment outcomes and can in some instances help patients and physicians to make more informed decisions regarding hepatitis C virus therapy."}

Genotyping of the ITPA variants allows the identification of a minority of patients who are highly unlikely to suffer from severe anemia during peg-IFN/RBV therapy. Given the necessary close follow-up of blood cell counts during treatment, this information will not be very relevant in standard clinical care, but could still be helpful in selected cases: for example, a treatment could possibly be started in a patient with serious cardioischemic or renal comorbidity if he has a protective ITPA genotype.

\section{Chances \& pitfalls of personalized HCV therapy}

The recent discoveries in the human genomics of HCV infection open new avenues in basic science and substantially improve our understanding of the natural history of the disease and of treatment outcomes. We can now better predict who will respond to HCV therapy and who will tolerate it. However, new knowledge brings new challenges to address, and pitfalls to avoid. First and foremost, it is essential to explain the nature and the relevance of the genetic findings to healthcare providers and patients: the genetic variants have strong effects at the population level, but are imperfect predictors at the individual level. Genotypes can be used to improve clinical decisions, but will not replace a global clinical 
assessment. It is also very important to avoid inappropriate denial of HCV therapy to individuals who would have responded to treatment despite carrying $I L 28 B$ risk alleles: health insurers and policy makers must understand the imperfect nature of the individual genetic prediction. Finally, there are still many open questions, and ongoing studies will hopefully help us get a better sense of the true relevance of $I L 28 B$ variation with regards to basic pathogenesis, non-1 HCV genotypes and novel combination therapies.

Hepatitis $\mathrm{C}$ just entered the pharmacogenomic era, and it is our strong belief that the discoveries discussed here create more opportunities than pitfalls. Experienced physicians are used to cautiously interpreting new research findings and will appropriately integrate new information into their treatment algorithms. This will improve treatment efficacy and tolerability - the ultimate goal of personalized hepatitis $\mathrm{C}$ therapy.

\section{Financial \& competing interests disclosure}

Andri Rauch and Jacques Fellay are co-applicants of patents that are currently being evaluated on the original finding of the IL28B association with spontaneous and treatment-induced HCV clearance. The authors have no other relevant affiliations or financial involvement with any organization or entity with a financial interest in or financial conflict with the subject matter or materials discussed in the manuscript apart from those disclosed.

No writing assistance was utilized in the production of this manuscript.

\section{References}

1 Thomas DL, Astemborski J, Rai RM et al. The natural history of hepatitis $\mathrm{C}$ virus infection: host, viral, and environmental factors. JAMA 284(4), 450-456 (2000).

2 Kau A, Vermehren J, Sarrazin C. Treatment predictors of a sustained virologic response in hepatitis $\mathrm{B}$ and $\mathrm{C}$. J. Hepatol. 49(4), 634-651 (2008).

3 Ge D, Fellay J, Thompson AJ et al. Genetic variation in IL28B predicts hepatitis C treatment-induced viral clearance. Nature 461(7262), 399-401 (2009).

4 Tanaka Y, Nishida N, Sugiyama M et al. Genome-wide association of $I L 28 B$ with response to pegylated interferon- $\alpha$ and ribavirin therapy for chronic hepatitis $\mathrm{C}$. Nat. Genet. 41(10), 1105-1109 (2009).

5 Suppiah V, Moldovan M, Ahlenstiel G et al. IL28B is associated with response to chronic hepatitis $\mathrm{C}$ interferon- $\alpha$ and ribavirin therapy. Nat. Genet. 41, 1100-1104 (2009).

6 Rauch A, Kutalik Z, Descombes P et al. Genetic variation in IL28B is associated with chronic hepatitis $\mathrm{C}$ and treatment failure: a genome-wide association study. Gastroenterology 138(4), 1338-1345 (2010).

7 Rauch A, Rohrbach J, Bochud PY. The recent breakthroughs in the understanding of host genomics in hepatitis C. Eur. J. Clin. Invest. 40(10), 950-959 (2010).
8 Thomas DL, Thio CL, Martin MP et al. Genetic variation in $I L 28 B$ and spontaneous clearance of hepatitis $C$ virus. Nature 461(7265), 798-801 (2009).

9 Thompson AJ, Muir AJ, Sulkowski MS et al. Interleukin-28B polymorphism improves viral kinetics and is the strongest pretreatment predictor of sustained virologic response in genotype 1 hepatitis $\mathrm{C}$ virus. Gastroenterology 139(1), 120-129 (2010).

10 Urban TJ, Thompson AJ, Bradrick SS et al. $I L 28 B$ genotype is associated with differential expression of intrahepatic interferon-stimulated genes in patients with chronic hepatitis C. Hepatology 52(6), 1888-1896 (2010).

11 Sarasin-Filipowicz M, Oakeley EJ, Duong $\mathrm{FH}$ et al. Interferon signaling and treatment outcome in chronic hepatitis $\mathrm{C}$. Proc. Natl Acad. Sci. USA 105(19), 7034-7039 (2008).

12 Fukuhara T, Taketomi A, Motomura T et al. Variants in $I L 28 B$ in liver recipients and donors correlate with response to peg-interferon and ribavirin therapy for recurrent hepatitis C. Gastroenterology 139(5), 1577-1585 (2010).

13 Medrano J, Neukam K, Rallon N et al. Modeling the probability of sustained virological response to therapy with pegylated interferon plus ribavirin in patients coinfected with hepatitis $C$ virus and HIV. Clin. Infect. Dis. 51(10), 1209-1216 (2010).
14 Akuta N, Suzuki F, Hirakawa M et al. Amino acid substitution in hepatitis $\mathrm{C}$ virus core region and genetic variation near the interleukin $28 \mathrm{~B}$ gene predict viral response to telaprevir with peginterferon and ribavirin. Hepatology 52(2), 421-429 (2010).

15 Thompson AJ, Muir AJ, Sulkowski MS et al. Hepatitis $\mathrm{C}$ trials that combine investigational agents with pegylated interferon should be stratified by interleukin-28B genotype. Hepatology 52(6) 2243-2244 (2010).

16 Rauch A, Egger M, Reichen J, Furrer H. Chronic hepatitis C in HIV-infected patients: low eligibility and applicability of therapy with pegylated interferon- $\alpha$ plus ribavirin. J. Acquir. Immune Defic. Syndr. 38(2), 238-240 (2005).

17 Bhattacharya D, Umbleja T, Carrat F et al. Women experience higher rates of adverse events during hepatitis $\mathrm{C}$ virus therapy in HIV infection: a meta-analysis. J. Acquir. Immune Defic. Syndr. 55(2), 170-175 (2010).

18 Thompson AJ, Fellay J, Patel K et al. Variants in the ITPA gene protect against ribavirin-induced hemolytic anemia and decrease the need for ribavirin dose reduction. Gastroenterology 139(4), 1181-1189 (2010).

19 Fellay J, Thompson AJ, Ge D et al. ITPA gene variants protect against anaemia in patients treated for chronic hepatitis C. Nature 464(7287), 405-408 (2010). 